\title{
Growth and smooth spectral synthesis in the Fourier algebras of Lie groups
}

\author{
by \\ JeAn Ludwig (Metz) and Lyudmila Turowska (Göteborg)
}

\begin{abstract}
Let $G$ be a Lie group and $A(G)$ the Fourier algebra of $G$. We describe sufficient conditions for complex-valued functions to operate on elements $u \in A(G)$ of certain differentiability classes in terms of the dimension of the group $G$. Furthermore, generalizing a result of Kirsch and Müller [Ark. Mat. 18 (1980), 145-155] we prove that closed subsets $E$ of a smooth $m$-dimensional submanifold of a Lie group $G$ having a certain cone property are sets of smooth spectral synthesis. For such sets we give an estimate of the degree of nilpotency of the quotient algebra $I_{A}(E) / J_{A}(E)$, where $I_{A}(E)$ and $J_{A}(E)$ are the largest and the smallest closed ideals in $A(G)$ with hull $E$.
\end{abstract}

1. Introduction. In this paper we study two questions concerning the Fourier algebra $A(G)$ of a (noncommutative) Lie group $G$. The first one deals with the functional calculus in $A(G)$ and the other with problems of spectral synthesis in that algebra.

Functional calculus is one of the basic tools in the theory of Banach algebras and in its applications. In particular, it plays a fundamental role in some parts of harmonic analysis, where one of the important algebras is the Fourier algebra $A(G)$ for a locally compact group $G$. One says that a complex-valued function $\varphi$ defined on $\mathbb{C}$ operates on $u \in A(G)$ if the composition $\varphi \circ u: G \rightarrow \mathbb{C}$ belongs to $A(G)$. It is known that any analytic function defined on a neighbourhood of the image of $u \in A(G)$ operates on $u$. Furthermore H. Helson, J.-P. Kahane, Y. Katznelson and W. Rudin [HKKR] for the case of $G$ abelian, and then C. Dunkl [D] and D. Rider [Ri] for infinite compact groups $G$, showed that exactly the real-analytic functions $\varphi: \mathbb{C} \rightarrow \mathbb{C}$ operate on each element of $A(G)$. Some necessary conditions on the functions $\varphi$ operating on certain classes of elements of $A(G)$ for abelian $G$ can be found for example in [Ka]. In order to determine the class $[u]$ of functions which operate on a given element $u$ of $A(G)$, it is essential to have control over the growth of the norms $\left\|e^{i t u}\right\|_{A(G)}$ for $t \in \mathbb{R}$ and real functions $u$

2000 Mathematics Subject Classification: Primary 46J10; Secondary 46E15, 43A45, 22E30. 
in $A(G)$. In this paper we study this question for Lie groups. First we give an estimate of the growth in $t$ of the norms $\left\|e^{i t u}\right\|_{A(G)}$ for a connected compact Lie group and real-valued functions $u$ of a certain differentiability class in terms of the dimension of $G$. Our method is a development of the method given in $[\mathrm{Ka}]$ for the one-dimensional torus. For a general Lie group $G$, we use a Laplacian and its heat kernel to derive for smooth functions $u$ on $G$ a similar estimate for $\left\|e^{i t u}\right\|_{A(G)}$. This result is slightly rougher than in the compact case. These estimates allow us to find necessary conditions for a function $\varphi$ to operate on smooth functions $u \in A(G)$.

Section 4 deals with questions of spectral synthesis in $A(G)$ for Lie groups $G$. It is well known that for any closed subset $E \subset G$ there exists a largest closed ideal $I_{A}(E)$ and a smallest closed ideal $J_{A}(E)$ with hull $E$. We say that $E$ is a set of spectral synthesis if $I_{A}(E)=J_{A}(E)$. If $E$ is not spectral, it may nevertheless happen that the algebra $I_{A}(E) / J_{A}(E)$ is nilpotent (see [W], where such sets $E$ are studied). We say that $E$ is a set of smooth spectral synthesis if $\overline{I_{A}(E) \cap \mathcal{D}(G)}=I_{A}(E)$, where $\mathcal{D}(G)$ is the set of test functions on $G$. Generalizing a method in $[\mathrm{KM}]$ we show that closed subsets $E$ of a smooth $m$-dimensional submanifold of a Lie group $G$ with a certain cone property are sets of smooth synthesis. Then using Herz's arguments $[\mathrm{H}]$ we obtain an estimate of the nilpotency degree of the algebra $I_{A}(E) / J_{A}(E)$.

In the last section we give some applications to linear operator equations and to Varopoulos algebras.

2. Preliminaries and notations. Let $G$ be a locally compact $\sigma$-compact separable group with left Haar measure $m=d g$. Let $L^{p}(G), p=1,2$, denote the space of $p$-integrable functions with norm $\|\cdot\|_{p}$ and let $C(G)$ denote the algebra of continuous complex-valued functions on $G$. The convolution algebra $L^{1}(G)$ is an involutive algebra with involution defined by $f^{*}(s)=\Delta^{-1}(s) f\left(s^{-1}\right)$, where $\Delta$ is the modular function of the group. Let $\lambda: G \rightarrow B\left(L^{2}(G)\right)$ be the left regular representation given by $\lambda(s) f(g)=$ $f\left(s^{-1} g\right)$. We denote by $\operatorname{VN}(G)$ the von Neumann algebra of $G$, that is,

$$
\mathrm{VN}(G)=\overline{\operatorname{span}}^{\mathrm{WOT}}\{\lambda(g): g \in G\} \subset B\left(L^{2}(G)\right) .
$$

The Fourier algebra is the family of functions $s \mapsto(\lambda(s) \xi, \eta)=\bar{\eta} * \check{\xi}$, $\xi, \eta \in L^{2}(G), \check{\xi}(s)=\xi\left(s^{-1}\right), s \in G$, as defined by Eymard in [E]. The Banach space $A(G)$ can be identified with the predual $\mathrm{VN}(G)_{*}$ via $\langle(\lambda(s) \xi, \eta), T\rangle=$ $(T \xi, \eta)$, and thus is a normed algebra with the norm denoted by $\|\cdot\|_{A}$. It is known that for $u \in A(G)$ there exist $\xi, \eta \in L^{2}(G)$ such that $u=\xi * \check{\eta}$ and $\|u\|_{A}=\|\xi\|_{2} \cdot\|\eta\|_{2}$. The algebra $A(G)$ is a semisimple regular Banach algebras with spectrum $G$ (see $[\mathrm{E}]$ ). 


\section{Growth in the Fourier algebra of a Lie group and functional calculus in $A(G)$}

3.1. The compact Lie case. The following description of the dual space of a connected compact Lie group $G$ has been taken from [Wa]. Let $\mathfrak{g}$ be the Lie algebra of $G$. Then $\mathfrak{g}=\mathfrak{z} \oplus \mathfrak{g}_{1}$ with $\mathfrak{z}$ the centre of $\mathfrak{g}$ and $\mathfrak{g}_{1}=[\mathfrak{g}, \mathfrak{g}]$ a compact Lie algebra. Let $\langle$,$\rangle be an inner product on \mathfrak{g}$ satisfying (1) $\left\langle\mathfrak{g}_{1}, \mathfrak{z}\right\rangle=(0)$ and $(2)\langle,\rangle_{\mid \mathfrak{g}_{1} \times \mathfrak{g}_{1}}=-B_{\mathfrak{g}_{1}}$ (here $B_{\mathfrak{k}}$ denotes the Killing form of a Lie algebra $\mathfrak{k})$. Let $U(\mathfrak{g})$ be the universal enveloping algebra of $\mathfrak{g}$. Let $X_{1}, \ldots, X_{n}$ be an orthonormal basis of $\mathfrak{g}$ such that $\left\{X_{1}, \ldots, X_{r}\right\}$ is a basis of $\mathfrak{z}$. Set $\Omega=\sum_{i} X_{i}^{2} \in U(\mathfrak{g})$. Then $\Omega$ is independent of the choice of the orthonormal basis of $\mathfrak{g}$ and $\Omega$ is central in $U(\mathfrak{g})$.

Let $\mathfrak{t}$ be a maximal abelian subalgebra of $\mathfrak{g}_{1}$ and let $T=\exp (\mathfrak{t})$. Let also $\lambda_{1}, \ldots, \lambda_{r}$ be complex-valued linear forms on $\mathfrak{z}$ defined by $\lambda_{j}\left(X_{i}\right)=$ $2 \pi(-1)^{1 / 2} \delta_{i, j}$. Let $P$ be a Weyl chamber of $T$. Let $\Lambda_{1}, \ldots, \Lambda_{l}$ be defined by $2 \Lambda_{i}\left(H_{\alpha_{j}}\right) / \alpha_{j}\left(H_{\alpha_{j}}\right)=\delta_{i, j}$, where $\alpha_{1}, \ldots, \alpha_{l}$ are the simple roots relative to $P$, and the $H_{\alpha_{j}}$ the corresponding vectors in $\mathfrak{t}$. To every $\gamma$ in the dual space $\widehat{G}$ of $G$ corresponds a unique element $\Lambda_{\gamma}=\sum_{i} n_{i} \lambda_{i}+\sum_{j} m_{j} \Lambda_{j}$ with the $n_{i}$ integers and the $m_{j}$ nonnegative integers. Set $\|\gamma\|=\max _{i, j}\left\{\left|n_{i}\right|, m_{j}\right\}$.

We know from [Wa, Lemma 5.6.4] (with the notations of that lemma) that for every $\gamma \in \widehat{G}$ and $\pi_{\gamma} \in \gamma$,

$$
-\pi_{\gamma}(\Omega)=\left(\left\langle\Lambda_{\gamma}+\varrho, \Lambda_{\gamma}+\varrho\right\rangle-\langle\varrho, \varrho\rangle\right) \mathbb{I}_{\mathcal{H}_{\gamma}}=: c(\gamma) \mathbb{I}_{\mathcal{H}_{\gamma}},
$$

where $\varrho$ is half the sum of the positive roots of $G$ related to the Weyl chamber of $T$. Then by [Wa, Lemma 5.6.6], there are positive constants $c_{1}, c_{2}$ such that

$$
c_{1}\|\gamma\|^{2} \leq c(\gamma) \leq c_{2}\|\gamma\|^{2}
$$

and by [Wa, Lemma 5.6.7], the series

$$
\sum_{\gamma \in \widehat{G}} d(\gamma)^{2}\left(1+\|\gamma\|^{2}\right)^{-s}
$$

converges if $s>\operatorname{dim}(G) / 2$. Here $d(\gamma)$ denotes the dimension of the Hilbert space $\mathcal{H}_{\gamma}$ of $\gamma$.

Also for a real number $a$, let $[a]$ be the integer part of $a$ and let $d(G)$ denote the dimension of the group $G$.

THEOREM 3.1. Let $G$ be a connected compact Lie group and let $u=\bar{u}$ be a self-adjoint element of $A(G)$, which is differentiable of class $C^{[d(G) / 2]+1}$. Then there exists a positive constant $C=C(u)$ such that

$$
\left\|e^{i t u}\right\|_{A(G)} \leq C(1+|t|)^{d(G) / 2}, \quad t \in \mathbb{R} .
$$


Proof. By (3.1) and (3.2), we know that

$$
\sum_{\gamma \in \widehat{G}} \frac{d(\gamma)^{2}}{(1+c(\gamma))^{s}}<\infty, \quad \forall s>d(G) / 2 .
$$

Take $N \in \mathbb{N}^{*}$ and let $\widehat{G}_{N}=\{\gamma \in \widehat{G}:\|\gamma\| \leq N\}$. By the Plancherel theorem, for all real $m>d(G) / 4$ there exists an $L^{2}$-function $E_{m}$ on $G$ such that

Then

$$
\gamma\left(E_{m}\right)=\frac{1}{(1+c(\gamma))^{m}} \mathbb{I}_{\mathcal{H}_{\gamma}}, \quad \forall \gamma \in \widehat{G} .
$$

$$
E_{m} *(1-\Omega)^{m} g=g
$$

for every $k$-times differentiable function $g$ on $G$, where $k=2 m$ if $2 m$ is an integer and $k=[2 m]+1$ otherwise. Denote also by $F_{N}$ the element in $L^{2}(G)$ for which

$$
\gamma\left(F_{N}\right)= \begin{cases}\mathbb{I}_{\mathcal{H}_{\gamma}}, & \forall \gamma \in \widehat{G}_{N} \\ 0, & \text { otherwise }\end{cases}
$$

and by $E_{m, N}$ the element in $L^{2}(G)$ for which

$$
\gamma\left(E_{m, N}\right)= \begin{cases}\gamma\left(E_{m}\right), & \forall \gamma \notin \widehat{G}_{N}, \\ 0, & \text { otherwise. }\end{cases}
$$

Write now $g_{t}$ for $e^{i t u}, t \in \mathbb{R}$. Since $u \in C^{[d(G) / 2]+1}$, we also have $g_{t} \in$ $C^{[d(G) / 2]+1}$. We decompose $g_{t}$ into

$$
g_{t}=a_{t, N}+b_{t, N}
$$

where $a_{t, N}$ and $b_{t, N}$ are defined by

$$
\gamma\left(a_{t, N}\right)=\left\{\begin{array}{ll}
\gamma\left(g_{t}\right), & \forall \gamma \in \widehat{G}_{N}, \\
0, & \text { otherwise, }
\end{array} \quad \gamma\left(b_{t, N}\right)= \begin{cases}0, & \forall \gamma \in \widehat{G}_{N}, \\
\gamma\left(g_{t}\right), & \text { otherwise. }\end{cases}\right.
$$

Then $a_{t, N}$ is a $C^{\infty}$-vector and so $b_{t, N}=g_{t}-a_{t, N}$ is of class $C^{[d(G) / 2]+1}$. By the definition of $F_{N}$, we have

Hence

$$
F_{N} * a_{t, N}=a_{t, N}
$$

$$
\left\|a_{t, N}\right\|_{A(G)} \leq\left\|F_{N}\right\|_{2}\left\|a_{t, N}\right\|_{2} \leq\left\|F_{N}\right\|_{2}\left\|g_{t}\right\|_{2} \leq\left\|F_{N}\right\|_{2}\left\|g_{t}\right\|_{L^{\infty}(G)} .
$$

Now, by [Wa, proof of 5.6.7], if we set $n=d(G)$ for simplicity, we have

$$
\left\|F_{N}\right\|_{2}^{2}=\sum_{\|\gamma\| \leq N} d_{\gamma}^{2} \leq c_{3} \sum_{j=0}^{N} j^{n-l-r}(2 r+l)(2 j+1)^{r+l-1} \leq c_{4} \sum_{j=0}^{N} j^{n-1} \leq c_{5} N^{n} .
$$

Hence, since $u$ is a continuous real-valued function, we have $g_{t}=e^{i t u} \in$ $L^{\infty}(G), t \in \mathbb{R}$, and $\left\|g_{t}\right\|_{\infty}=1$, and we see that

$$
\left\|a_{t, N}\right\|_{A(G)} \leq C N^{d(G) / 2}
$$


for a certain constant $C>0$. Now for the norm of the element $b_{t, N}$ we get, using (3.5) for $g=b_{t, N}$ and $m=\frac{1}{2}([d(G) / 2]+1$ ) (which is easily checked to be strictly larger than $d(G) / 4)$,

$$
E_{m} *(1-\Omega)^{m} b_{t, N}=E_{m, N} *(1-\Omega)^{m} g_{t}
$$

and so

$$
\left\|b_{t, N}\right\|_{A(G)}=\left\|E_{m, N} *(1-\Omega)^{m} g_{t}\right\|_{A(G)} \leq\left\|E_{m, N}\right\|_{2}\left\|(1-\Omega)^{m} g_{t}\right\|_{2} .
$$

If $m$ is an integer then, since $\Omega$ is a differential operator of order 2 , we have $(1-\Omega)^{m} g_{t}=\left(1+t^{2 m}\right) e^{i t u} w_{t, m}$ for some $w_{t, m} \in L^{2}(G)$ such that $\left\|w_{t, m}\right\|_{2} \leq C_{1}$ for all $t \in \mathbb{R}$ (for some constant $C_{1}>0$ ). If $m$ is a half integer $k+1 / 2$ then we use the inequality

$$
\left\|(1-\Omega)^{1 / 2} \eta\right\|_{2} \leq\left(\|\eta\|_{2}^{2}+\sum_{j}\left\|X_{j} \eta\right\|_{2}^{2}\right)^{1 / 2}
$$

for any differentiable $\eta$. Indeed, if $\eta$ is twice differentiable, then

$$
\left\|(1-\Omega)^{1 / 2} \eta\right\|_{2}^{2}=\langle(1-\Omega) \eta, \eta\rangle=\|\eta\|_{2}^{2}-\sum_{j}\left\langle X_{j}^{2} \eta, \eta\right\rangle=\|\eta\|_{2}^{2}+\sum_{j}\left\|X_{j} \eta\right\|_{2}^{2} .
$$

We can now approximate a once differentiable $\eta$ with respect to the Sobolev norm $\|\eta\|_{S}:=\left(\|\eta\|_{2}^{2}+\sum_{j}\left\|X_{j} \eta\right\|_{2}^{2}\right)^{1 / 2}$ by twice differentiable functions to obtain our inequality (3.6). Therefore, for $\eta_{t}:=(1-\Omega)^{k} g_{t}$,

$$
\begin{aligned}
\left\|(1-\Omega)^{k+1 / 2} g_{t}\right\|_{2}^{2} & =\left\|(1-\Omega)^{1 / 2} \eta_{t}\right\|_{2}^{2} \\
& \leq\left\|\eta_{t}\right\|_{2}^{2}+\sum_{j}\left\|X_{j} \eta_{t}\right\|_{2}^{2} \\
& =\left\|(1-\Omega)^{k} g_{t}\right\|_{2}^{2}+\sum_{j}\left\|X_{j}(1-\Omega)^{k} g_{t}\right\|_{2}^{2} \\
& =\left\|\left(1+t^{2 k}\right) e^{i t u} v_{t, k}\right\|_{2}^{2}+\left\|\left(1+t^{2 k+1}\right) e^{i t u} w_{t, k}\right\|_{2}^{2}
\end{aligned}
$$

for some $w_{t, k}, v_{t, k} \in L^{2}(G)$ with $L^{2}$-norms uniformly bounded in $t$. Hence

$$
\left\|(1-\Omega)^{m} g_{t}\right\|_{2} \leq C\left(1+|t|^{2 m}\right), \quad t \in \mathbb{R},
$$

for some new constant $C>0$ and for $m=k+1 / 2$.

Therefore we get the following estimate of the $A(G)$-norm of $b_{t, N}$ : for $t \in \mathbb{R}$,

$$
\begin{aligned}
\left\|b_{t, N}\right\|_{A(G)} & \leq C_{1}\left(\sum_{\|\gamma\|>N} \frac{d(\gamma)^{2}}{(1+c(\gamma))^{2 m}}\right)^{1 / 2}(1+|t|)^{2 m} \\
& \leq C_{2}\left(\sum_{j>N} j^{n-1} \frac{1}{(1+j)^{4 m}}\right)^{1 / 2}(1+|t|)^{2 m} \leq C_{3} \frac{1}{N^{2 m-n / 2}}(1+|t|)^{2 m}
\end{aligned}
$$


Hence, if we let $N$ be the smallest integer $\geq|t|$ we obtain

$$
\begin{aligned}
\left\|e^{i t u}\right\|_{A(G)} & \leq\left\|a_{t, N}\right\|_{A(G)}+\left\|b_{t, N}\right\|_{A(G)} \\
& \leq \frac{C}{2}(1+|t|)^{d(G) / 2}+\frac{C}{2}(1+|t|)^{d(G) / 2}
\end{aligned}
$$

for a new constant $C>0$.

REMARK 3.2. In the case of the one-dimensional torus $G=\mathbb{T}$ it is shown in $[\mathrm{Ka}]$ that if $u=\bar{u} \neq$ const is differentiable of class $C^{2}$, the estimate (3.3) is sharp, i.e. then $\left\|e^{i n u}\right\|_{A(\mathbb{T})} \approx \sqrt{n}$. We could also ask for which class of functions $u=\bar{u} \in A(G)$ on a compact Lie group $G$ we can have $\left\|e^{i t u}\right\|_{A(\mathbb{T})} \approx t^{d(G) / 2}$.

3.2. The case of a general nondiscrete Lie group. We now consider a Lie group $G$ of positive dimension and we let $G_{0}$ be its connected component. Choose a basis $\left\{X_{1}, \ldots, X_{n}\right\}$ of the Lie algebra $\mathfrak{g}$ of $G$ and take again a Laplacian $\Omega=\sum_{i=1}^{n} X_{i}^{2}$ on $G$. Let furthermore $\left(h_{t}\right)_{t>0}$ be the heat kernel on $G_{0}$ associated to $\Omega$ (see [VSC] for the definition).

Define the function $E_{m}(m \in \mathbb{N}, m>d(G) / 4)$ on $G_{0}$ by the formula

$$
E_{m}=\frac{1}{m !} \int_{0}^{\infty} t^{m-1} e^{-t} h_{t} d t
$$

The function $E_{m}$ is clearly in $L^{1}\left(G_{0}\right) \subset L^{1}(G)$ and $\int_{G} E_{m}(g) d g=1$, since $\left\|h_{t}\right\|_{1}=\int_{G} E_{m}(g) d g=1$ for all $t>0$.

Furthermore, denoting by $\lambda$ the left regular representation of $G$ on $L^{2}(G)$, we find that $\lambda\left(E_{m}\right)$ is the inverse of the operator $(1-\Omega)^{m}$. In fact, let

$$
-\Omega=\int_{0}^{\infty} s d P_{s}
$$

be the spectral resolution of $-\Omega$. Then

$$
(1-\Omega)^{m}=\int_{0}^{\infty}(1+s)^{m} d P_{s}, \quad \lambda\left(h_{t}\right)=e^{t \Omega}=\int_{0}^{\infty} e^{-t s} d P_{s}
$$

by the definition of the heat kernel and

$$
\begin{aligned}
\lambda\left(E_{m}\right) & =\frac{1}{m !} \int_{0}^{\infty} t^{m-1} e^{-t} \int_{0}^{\infty} e^{-t s} d P_{s} d t \\
& =\frac{1}{m !} \int_{0}^{\infty} \int_{0}^{\infty} t^{m-1} e^{-(1+s) t} d t d P_{s} \\
& =\frac{1}{m !} \int_{0}^{\infty} \frac{1}{(1+s)^{m}} m ! d P_{s}=(1-\Omega)^{-m}
\end{aligned}
$$


This means in particular that

$$
E_{m} *(1-\Omega)^{m} f=f
$$

for any $f \in \mathcal{D}(G)$, the space of compactly supported $C^{\infty}$-functions on $G$.

For the estimation of the $A(G)$ norm, we must show that the functions $\check{E}_{m}$ defined by $\check{E}_{m}(g):=E_{m}\left(g^{-1}\right), g \in G$, are in $L^{2}(G)$ for $m>d(G) / 4$.

LEMMA 3.3. There exists a Laplacian $\Omega$ on $G$ such that the functions $\check{E}_{m}$ are in $L^{2}(G)$ for $m>d(G) / 4$.

Proof. If $G_{0}$ is not unimodular, let $G_{1}$ be the kernel of the modular function $\Delta$ in $G_{0}$. Since $\Delta(\exp (t U))=e^{-t \delta(U)}, t \in \mathbb{R}, U \in \mathfrak{g}$, where $\delta: \mathfrak{g} \rightarrow \mathbb{R}$ denotes the trace of $\operatorname{ad}(U)$, it follows that $G_{1}$ is of codimension one in $G$ and that $G$ is the topological product of $\mathbb{R}$ and $G_{1}$, since now the function $\delta$ is not 0 . Let $\mathfrak{g}_{1}=\operatorname{ker}(\delta)$ be the Lie algebra of $G_{1}$. We choose a basis vector $X=X_{1}$ in $\mathfrak{g} \backslash \mathfrak{g}_{1}$ and vectors $X_{2}, \ldots, X_{n}$ in $\mathfrak{g}_{1}$. We can also assume that

$$
\delta(X)=-1 / 2, \quad \text { i.e. } \quad \Delta(\exp (s X))=e^{s / 2}, \quad s \in \mathbb{R} .
$$

It is easy to see then that

$$
\Delta(g) \leq e^{d \varrho(g)}, \quad g \in G,
$$

where $\varrho(g)$ denotes the Carnot-Carathéodory distance of $g$ to the origin and where $d$ is some positive constant (see [VSC]).

Since the linear operators $\lambda\left(h_{t}\right), t>0$, are self-adjoint and the functions $h_{t}$ are real-valued, it follows that

$$
\check{h}_{t}(g)=h_{t}\left(g^{-1}\right)=\Delta(g) h_{t}(g)
$$

for every $g \in G$. Hence by $(3.7), \check{E}_{m}=\Delta E_{m}$. By [VSC, V.4.3 and IX.1.3] and (3.10), it follows that for some constant $C>0$,

$$
\Delta(g) h_{t}(g) \leq C \frac{1}{t^{d(G) / 2}}, \quad 0<t \leq \varepsilon, g \in G
$$

for some constant $\varepsilon>0$.

Define for $t>0$ the function $p_{t}$ on $\mathbb{R}$ by

$$
p_{t}(s):=\int_{G_{1}} h_{t}\left(\exp (s X) g_{1}\right) d g_{1}, \quad s \in \mathbb{R} .
$$

The functions $p_{t}$ are then of class $C^{\infty}$ and they satisfy the equations

$$
\begin{aligned}
X_{j} p_{t}(s) & =\int_{G_{1}} X_{j} h_{t}\left(\exp (s X) g_{1}\right) d g_{1} \\
& =\int_{G_{1}} \frac{d}{d u} h_{t}\left(\exp \left(-u X_{j}\right) \exp (s X) g_{1}\right)_{u=0} d g_{1}
\end{aligned}
$$




$$
\begin{aligned}
& =\int_{G_{1}} \frac{d}{d u} h_{t}\left(\exp (s X) \exp \left(-u \operatorname{Ad}(\exp (-s X)) X_{j}\right) g_{1}\right)_{u=0} d g_{1} \\
& =\int_{G_{1}} \frac{d}{d u} h_{t}\left(\exp (s X) g_{1}\right)_{u=0} d g_{1}=0
\end{aligned}
$$

for $j=2, \ldots, n$, and therefore

$$
\begin{aligned}
\left(\frac{\partial}{\partial s}\right)^{2} p_{t}(s) & =\int_{G_{1}} X^{2} h_{t}\left(\exp (s X) g_{1}\right) d g_{1}=\int_{G_{1}} \Omega h_{t}\left(\exp (s X) g_{1}\right) d g_{1} \\
& =\int_{G_{1}} \frac{\partial}{\partial t} h_{t}\left(\exp (s X) g_{1}\right) d g_{1}=\frac{\partial}{\partial t} p_{t}(s) .
\end{aligned}
$$

Hence $p_{t}$ is the heat kernel on $\mathbb{R}$ and so $p_{t}(s)=(c / \sqrt{t}) e^{-s^{2} / 4 t}, s \in \mathbb{R}$ (for some $c>0$ ). This shows that

$$
\begin{aligned}
\left\|\Delta h_{t}\right\|_{1} & =\int_{G} \Delta(g) h_{t}(g) d g \\
& =\int_{\mathbb{R}} \Delta(\exp (s X)) \int_{G_{1}} h_{t}\left(\exp (s X) g_{1}\right) d g_{1} d s \\
& =\int_{\mathbb{R}} e^{s / 2} p_{t}(s) d s \quad(\text { by } 3.9) \\
& =\int_{\mathbb{R}} e^{s / 2} c \frac{1}{\sqrt{t}} e^{-s^{2} / 4 t} d s=c^{\prime} e^{t / 4}, \quad t>0
\end{aligned}
$$

for some new $c^{\prime}>0$.

From the inequalities (3.12) and (3.14) we deduce that for a certain constant $C>0$,

$$
\left.\left.\left\|\Delta h_{t}\right\|_{2} \leq \sqrt{\left\|\Delta h_{t}\right\|_{\infty}} \sqrt{\left\|\Delta h_{t}\right\|_{1}} \leq C \frac{1}{t^{d(G) / 4}}, \quad t \in\right] 0, \varepsilon\right],
$$

and if $t>\varepsilon$, then $\Delta h_{t}=\Delta h_{t-\varepsilon / 2} * \Delta h_{\varepsilon / 2}$ and so

$$
\left\|\Delta h_{t}\right\|_{2}=\left\|\Delta h_{t-\varepsilon / 2} * \Delta h_{\varepsilon / 2}\right\|_{2} \leq\left\|\Delta h_{t-\varepsilon / 2}\right\|_{1}\left\|\Delta h_{\varepsilon / 2}\right\|_{2} \leq D e^{t / 4} .
$$

Hence, for $m>d(G) / 4$,

$$
\begin{aligned}
\left\|\check{E}_{m}\right\|_{2} & =\left\|\Delta E_{m}\right\|_{2}=\frac{1}{m !}\left\|\int_{\mathbb{R}} t^{m} e^{-t} \Delta h_{t} d t\right\|_{2} \\
& \leq \frac{1}{m !} \int_{0}^{\varepsilon} t^{m} \frac{1}{t^{d(G) / 4}} d t+\frac{1}{m !} \int_{\varepsilon}^{\infty} t^{m} D e^{-3 t / 4} d t<\infty .
\end{aligned}
$$

The unimodular case is similar, but easier.

We can now prove the following theorem. 
Theorem 3.4. Let $u=\bar{u}$ be a self-adjoint element of $A(G) \cap L^{2}(G)$ which is differentiable of class $C^{2([d(G) / 4]+1)}$ with bounded partial derivatives contained in $L^{2}(G)$. Then there exists a positive constant $C=C(u)$ such that

$$
\left\|e^{i t u}-1\right\|_{A(G)} \leq C(1+|t|)^{2[d(G) / 4]+2}, \quad t \in \mathbb{R} .
$$

Proof. Let $m=[d(G) / 4]+1$. As in the proof of Theorem 3.1, we see that

$$
\begin{aligned}
e^{i t u} & =E_{m} *(1-\Omega)^{m}\left(e^{i t u}\right) \\
& =E_{m} *\left(e^{i u t}+\left(1+|t|^{2 m}\right) e^{i u t} w_{t, m}\right) \\
& =E_{m} *\left(1+i t u\left(\frac{e^{i t u}-1}{i t u}\right)+\left(1+|t|^{2 m}\right) e^{i u t} w_{t, m}\right) \\
& =E_{m} * 1+t E_{m} *\left(i u\left(\frac{e^{i t u}-1}{i t u}\right)\right)+\left(1+|t|^{2 m}\right) E_{m} *\left(e^{i u t} w_{t, m}\right) \\
& =1+\left(1+|t|^{2 m}\right) E_{m} * v_{t, m}
\end{aligned}
$$

for some $w_{t, m}, v_{t, m} \in L^{2}(G)$ whose $L^{2}(G)$ norms are bounded by some constant $C>0$.

Hence using (3.18), we obtain

$$
\left\|e^{i t u}-1\right\|_{A(G)} \leq(1+|t|)^{2 m}\left\|\check{E}_{m}\right\|_{2}\left\|v_{t, m}\right\|_{2} .
$$

Thus we get

$$
\left\|e^{i t u}-1\right\|_{A(G)} \leq C(1+|t|)^{2([d(G) / 4]+1)}, \quad t \in \mathbb{R},
$$

for some constant $C>0$.

Remark 3.5. Let $G$ be a discrete group. Then the Hilbert space $l^{2}(g)$ is contained in $A(G)$ and for every $u \in l^{2}(G)$ we have $\|u\|_{A(G)}=\|\delta * u\|_{A(G)} \leq$ $\|\delta\|_{2}\|u\|_{2}=\|u\|_{2}$ (here $\delta$ denotes the identity of $l^{1}(G)$ ). Hence if $u=\bar{u}$ is an element of $l^{2}(G) \subset A(G)$ then

$$
\left\|e^{i t u}-1\right\|_{A(G)} \leq C(1+|t|), \quad t \in \mathbb{R}
$$

for some constant $C>0$. Indeed,

$$
\begin{aligned}
\left\|e^{i t u}-1\right\|_{A(G)} & =\left\|i t u\left(\frac{e^{i t u}-1}{i t u}\right)\right\|_{A(G)} \\
& \leq(1+|t|)\left\|\frac{e^{i t u}-1}{i t u}\right\|_{\infty}\|u\|_{2}, \quad t \in \mathbb{R} .
\end{aligned}
$$

Corollary 3.6.

(a) Let $G$ be a connected compact Lie group. Let $u=\bar{u}$ be a self-adjoint element of $A(G)$ which is differentiable of class $C^{[d(G) / 2]+1}$. Then 
every function $\varphi$ of class $C^{m}$ with $m>[d(G) / 2]+1$, vanishing at 0 , operates on $u$.

(b) Let $G$ be a Lie group of positive dimension. Let $u=\bar{u}$ be a selfadjoint element of $A(G) \cap L^{2}(G)$ which is differentiable of class $C^{2([d(G) / 4]+1)}$ with bounded partial derivatives contained in $L^{2}(G)$. Then every function $\varphi$ of class $C^{m}$ with $m>2[d(G) / 4]+3$, vanishing at 0 , operates on $u$.

Proof. (a) Let $u=\bar{u} \in A(G)$ as in Theorem 3.1. Let $\varphi: \mathbb{R} \rightarrow \mathbb{C}$ be of class $C^{m}$ with $m>d(G) / 2+1$ such that $\varphi(0)=0$. Let $\varphi_{0}$ be any $C^{m}$ function on $\mathbb{R}$ with compact support such that $\varphi_{0}=\varphi$ on the interval $\left[-\|u\|_{A(G)}-1\right.$, $\left.\|u\|_{A(G)}+1\right]$. Then the Fourier transform $\widehat{\varphi}_{0}(t)=\int_{\mathbb{R}} \varphi_{0}(x) e^{-2 i \pi t x} d x, t \in \mathbb{R}$, of $\varphi_{0}$ satisfies the inequality

$$
\left|\widehat{\varphi}_{0}(t)\right| \leq \frac{C_{\varphi_{0}}}{(1+|t|)^{m}}, \quad t \in \mathbb{R},
$$

where $C_{\varphi_{0}}$ is some constant depending on $\varphi_{0}$. Therefore by (3.3) the integral

$$
v:=\int_{\mathbb{R}} \widehat{\varphi}_{0}(t) e^{i 2 \pi t u} d t
$$

converges in $A(G)$, hence also in $C(G)$, and so for every $s \in G$, by the Fourier inversion formula we have

$$
v(s)=\int_{\mathbb{R}} \widehat{\varphi}_{0}(t) e^{2 i \pi t u(s)} d t=\varphi_{0}(u(s))=\varphi(u(s)) .
$$

Hence $\varphi \circ u=v \in A(G)$. The proof of (b) is similar.

4. Smooth synthesis. Let $A$ be a semisimple, regular, commutative Banach algebra with $X_{A}$ as spectrum; for any $a \in A$ we denote by $\widehat{a} \in$ $C_{0}\left(X_{A}\right)$ its Gelfand transform. Let also $E \subset X_{A}$ be a closed subset. We then define

$$
\begin{gathered}
I_{A}(E)=\left\{a \in A: \widehat{a}^{-1}(0) \text { contains } E\right\} \\
J_{A}^{0}(E)=\left\{a \in A: \widehat{a}^{-1}(0) \text { contains a nbhd of } E\right\}, \quad J_{A}(E)=\overline{J_{A}^{0}(E)} .
\end{gathered}
$$

It is known that $I_{A}(E)$ and $J_{A}(E)$ are the largest and the smallest closed ideals with $E$ as hull, i.e., if $I$ is a closed ideal such that $\left\{x \in X_{A}: f(x)=0\right.$ for all $f \in I\}=E$ then

$$
J_{A}(E) \subset I \subset I_{A}(E) .
$$

We say that $E$ is a set of spectral synthesis for $A$ if $J_{A}(E)=I_{A}(E)$, and of weak synthesis if $I_{A}(E)^{d}=J_{A}(E)$ for some integer $d$ (see [W]).

Let $A^{*}$ be the dual of $A$. For $a \in A$ we set $\operatorname{supp}(a)=\overline{\left\{x \in X_{A}: \widehat{a}(x) \neq 0\right\}}$ and $\operatorname{null}(a)=\left\{x \in X_{A}: \widehat{a}(x)=0\right\}$. For $\tau \in A^{*}$ and $a \in A$ define $a \tau$ in $A^{*}$ 
by $a \tau(b)=\tau(a b)$ and define the support of $\tau$ by

$$
\operatorname{supp}(\tau)=\left\{x \in X_{A}: a \tau \neq 0 \text { whenever } \widehat{a}(x) \neq 0\right\} .
$$

It is known that $\operatorname{supp}(\tau)$ consists of all $x \in X_{A}$ such that for any neighbourhood $U$ of $x$ there exists $a \in A$ for which $\operatorname{supp}(a) \subset U$ and $\tau(a) \neq 0$. Then, for every closed set $E \subset X_{A}$,

$$
J_{A}(E)^{\perp}=\left\{\tau \in A^{*}: \operatorname{supp}(\tau) \subset E\right\}
$$

and $E$ is spectral for $A$ if and only if $\tau(a)=0$ for any $a \in A$ and $\tau \in A^{*}$ such that $\operatorname{supp}(\tau) \subset E \subset \operatorname{null}(a)$.

In what follows we write $I_{A}(E)$ for $I_{A(G)}(E)$ and $J_{A}(E)$ for $J_{A(G)}(E)$.

Let $G$ be a Lie group and let $\mathcal{D}(G)$ be the space of all compactly supported $C^{\infty}$-functions on $G$. For a closed subset $E$ of $G$, we denote by $J_{\mathcal{D}}(E)$ the space of all elements of $\mathcal{D}(G)$ which vanish on $E$. Let

$$
B(E):=\{\lambda(\mu): \mu \text { a bounded measure on } G \text { supported in } E\} .
$$

Here $\lambda(\mu) f=\mu * f$ denotes the convolution of the function $f$ with the measure $\mu$.

The closure of $B(E)$ is the annihilator of $I_{A}(E)$ in $\mathrm{VN}(G)$, where the closure is taken in the weak* topology $\sigma(\mathrm{VN}(G), A(G))$.

Definition 4.1. The closed subset $E$ of $G$ is said to be of smooth synthesis if $\overline{J_{\mathcal{D}}(E)}=I_{A}(E)$.

Let us remark that in the papers of Müller $[\mathrm{M}]$ and Guo $[\mathrm{G}]$ the word "weak" is used instead of "smooth".

Remark 4.2. Let $G$ be a Lie group and $f$ an element in $\mathcal{D}(G)$. Then by (3.8), for any integer $m>d(G) / 4$ we have $f=E_{m} * \check{g}$, where $g=$ $\left((1-\Omega)^{m} f\right)^{\sim} \in \mathcal{D}(G)$. Here $\check{u}(x):=u\left(x^{-1}\right)$ for any function $u$ on $G$ and $x \in G$. Hence, for $t \in G$, denoting by $\varrho(t)$ the right translation by $t$, we have

$$
\begin{aligned}
\varrho(t) f(x) & =f(x t)=\int_{G} E_{m}(u) g\left(t^{-1} x^{-1} u\right) d u \\
& =\int_{G} E_{m}(u) \lambda(t) g\left(x^{-1} u\right) d u=E_{m} *(\lambda(t) g)^{\curlyvee}(x) .
\end{aligned}
$$

This shows that the mapping $t \mapsto \varrho(t) f$ from $G$ to the Banach space $A(G)$ is smooth.

TheOREM 4.3. Let $G$ be a Lie group of dimension $n$. Let $M$ be a smooth submanifold of dimension $m<n$ and let $E$ be a compact subset of $M$. Then ${\overline{J_{\mathcal{D}}(E)}}^{[m / 2]+1}=J_{A}(E)$.

Proof. It suffices to show that $J_{\mathcal{D}}(E)^{[m / 2]+1} \subset J_{A}(E)$. Let $f \in J_{\mathcal{D}}(E)$. We will use first a procedure given in $[\mathrm{H}]$ to estimate the distance between 
$f^{[m / 2]+1}$ and $J_{A}(E)$. For $0<\varepsilon<\|f\|_{\infty}$ let

$$
\begin{aligned}
W_{\varepsilon} & =\left\{x \in G:\|\varrho(x) f-f\|_{A(G)}<\varepsilon\right\} \\
& \subset \Omega_{\varepsilon}=\left\{x \in G:\|\varrho(x) f-f\|_{\infty}<\varepsilon\right\} .
\end{aligned}
$$

Since $f$ is a $C^{\infty}$-function with compact support, the mapping $g \mapsto$ $\varrho(g) f \in A(G)$ is $C^{\infty}$ by Remark 4.2. Therefore there exist a constant $K>0$ and an open neighbourhood $W$ of 0 in the Lie algebra $\mathfrak{g}$ of $G$ such that

$$
\|\varrho(\exp (X)) f-f\|_{A(G)} \leq K\|X\|
$$

for every $X \in W$ and some fixed norm \|\| on $\mathfrak{g}$. For $\varepsilon>0$, let $V_{\varepsilon}=\exp \left(B_{\varepsilon}\right)$, where $B_{\varepsilon}$ denotes the ball of radius $\varepsilon / 2 K$ of centre 0 in $\mathfrak{g}$. For $\varepsilon$ small enough we have $B_{\varepsilon} \subset W$. There exist constants $C_{1}>C_{2}>0$ such that for every $\varepsilon>0$,

$$
C_{1} \varepsilon^{n}>\left|V_{\varepsilon}\right|>C_{2} \varepsilon^{n}
$$

and $V_{\varepsilon} \subset W_{\varepsilon} \subset \Omega_{\varepsilon}$. In particular, for every $x=x_{0} v \in E V_{\varepsilon}, x_{0} \in E, v \in V_{\varepsilon}$, we have $f\left(x_{0}\right)=0$ since $f \in J_{\mathcal{D}}(E)$, and therefore

$$
\begin{aligned}
|f(x)| & =\left|f\left(x_{0} v\right)\right| \leq\left|f\left(x_{0} v\right)-f\left(x_{0}\right)\right|+\left|f\left(x_{0}\right)\right| \\
& =\left|(\varrho(v) f-f)\left(x_{0}\right)\right| \leq\|(\varrho(v) f-f)\|_{\infty}<\varepsilon .
\end{aligned}
$$

Hence

$$
\left|f^{[m / 2]+1}(x)\right| \leq \varepsilon^{[m / 2]+1} .
$$

Take $\nu=f^{[m / 2]+1}$ on $E V_{\varepsilon}$ and $\nu=0$ elsewhere and let $u \in L^{2}(G), u \geq 0$, be such that $\int_{G} u(x) d x=1$ and $\operatorname{supp}(u) \subset V_{\varepsilon}$. Consider now the function

$$
\varphi(s)=\left(f^{[m / 2]+1}-\nu\right) * \check{u}(s)=\int_{G}\left(f^{[m / 2]+1}-\nu\right)(s t) u(t) d t .
$$

Clearly $\varphi \in A(G)$, and $\varphi(s)=0$ if $s \cdot \operatorname{supp}(u) \subset E V_{\varepsilon}$. Since $E \subset\{s$ : $\left.s \cdot \operatorname{supp}(u) \subset E V_{\varepsilon}\right\}$ and the set $\left\{s: s \cdot \operatorname{supp}(u) \subset E V_{\varepsilon}\right\}$ is open, $\operatorname{supp}(\varphi)$ is disjoint from $E$ and therefore $\varphi \in J_{A}(E)$. We have

$$
f^{[m / 2]+1}-\varphi=\left(f^{[m / 2]+1}-f^{[m / 2]+1} * \check{u}\right)+\nu * \check{u} .
$$

As $\operatorname{supp}(u) \subset V_{\varepsilon} \subset W_{\varepsilon}$, and $\left\|f^{[m / 2]+1}-\varrho(x) f^{[m / 2]+1}\right\|_{A(G)} \leq K \varepsilon$ for all $x \in W_{\varepsilon}$ and some constant $K=K(m)>0$ which is independent of $\varepsilon$, it follows that

$$
\begin{aligned}
\left\|f^{[m / 2]+1}-f^{[m / 2]+1} * \check{u}\right\|_{A(G)} & =\left\|\int_{G}\left(f^{[m / 2]+1}-\varrho(x) f^{[m / 2]+1}\right) u(x) d x\right\|_{A(G)} \\
& \leq \int_{G}\left\|f^{[m / 2]+1}-\varrho(x) f^{[m / 2]+1}\right\|_{A(G)} u(x) d x \leq K \varepsilon .
\end{aligned}
$$

We also have $\|\nu * \check{u}\|_{A(G)} \leq\|\nu\|_{2} \cdot\|u\|_{2}$. As the greatest lower bound for $\|u\|_{2}$ 
is $\left|V_{\varepsilon}\right|^{-1 / 2}$, we obtain

$$
\begin{aligned}
\operatorname{dist}\left(f^{[m / 2]+1}, J_{A}(E)\right) & \leq\left\|f^{[m / 2]+1}-\varphi\right\|_{A(G)} \\
& \leq K \varepsilon+\left|V_{\varepsilon}\right|^{-1 / 2}\left(\int_{E V_{\varepsilon}}\left|f^{[m / 2]+1}(x)\right|^{2} d x\right)^{1 / 2} \\
& \leq K \varepsilon+\left|V_{\varepsilon}\right|^{-1 / 2} \sup _{x \in E V_{\varepsilon}}\left|f^{[m / 2]+1}(x)\right|\left|E V_{\varepsilon}\right|^{1 / 2} \\
& \leq K \varepsilon+\frac{\varepsilon^{-n / 2}}{C_{2}^{1 / 2}} \varepsilon^{[m / 2]+1}\left|E V_{\varepsilon}\right|^{1 / 2} .
\end{aligned}
$$

Let us estimate $\left|E V_{\varepsilon}\right|$. Since $E$ is compact, we can assume that $E V_{\varepsilon}$ is covered by a finite number of charts and hence is contained in one chart, $\psi: U \rightarrow \mathbb{R}^{m} \times \mathbb{R}^{n-m}$, such that $\psi(M \cap U) \subset \mathbb{R}^{m} \times\{0\}$. Furthermore, there exists a constant $C>0$ and a bounded set $B$ in $\mathbb{R}^{m}$, which do not depend on $\varepsilon$, and a rectangle $R_{\varepsilon}$ of measure $C \varepsilon^{n-m}$ in $\mathbb{R}^{n-m}$ such that $\psi\left((M \cap U) V_{\varepsilon}\right) \subset B \times R_{\varepsilon}$. Since there exists a continuous positive function $F: U \rightarrow \mathbb{R}$ such that

$$
\int_{U} \varphi(g) d g=\int_{\psi(U)} F(x) \varphi\left(\psi^{-1}(x)\right) d x, \quad \varphi \in C_{\mathrm{c}}(U),
$$

we get, for every small $\varepsilon$,

$$
\left|E V_{\varepsilon}\right|=\int_{\psi\left(E V_{\varepsilon}\right)} F(x) d x \leq \int_{B \times R_{\varepsilon}} F(x) d x \leq C^{\prime} \varepsilon^{n-m}
$$

Hence, for $\varepsilon>0$ small enough,

$$
\begin{aligned}
\operatorname{dist}\left(f^{[m / 2]+1}, J_{A}(E)\right) & \leq K \varepsilon+C^{\prime \prime} \varepsilon^{-n / 2} \varepsilon^{[m / 2]+1} \varepsilon^{(n-m) / 2} \\
& =K \varepsilon+C^{\prime \prime} \varepsilon^{[m / 2]+1-m / 2}
\end{aligned}
$$

for a new constant $C^{\prime \prime}$ which does not depend on $\varepsilon$. Thus $f^{[m / 2]+1} \in J_{A}(E)$. It now follows from standard arguments that $J_{\mathcal{D}}(E)^{[m / 2]+1} \subset J_{A}(E)$.

COROLlARY 4.4. Let $E$ be a compact subset of a smooth $m$-dimensional submanifold of the Lie group $G$. If $E$ is a set of smooth synthesis, then $E$ is of weak synthesis with $I_{A}(E)^{[m / 2]+1}=J_{A}(E)$.

\section{Definition 4.5.}

1. Let $G$ be a group and let $\operatorname{Aut}(G)$ be the group of automorphisms of $G$. Let $t \in G$ and $a \in \operatorname{Aut}(G)$. We call the mapping $G \rightarrow G, s \mapsto a(t s)$, an affine transformation of $G$.

2. Let $G$ be a Lie group. We say that a group $A$ is a group of affine transformations of $G$ if $A$ is a Lie group which acts smoothly by affine transformations on $G$. Smoothly here means that the mapping $A \times G \rightarrow G,(a, x) \mapsto a(x)$, is smooth. 
Definition 4.6. Let $G$ be a Lie group and let $M$ be a smooth $m$ dimensional submanifold of $G$. We say that a subset $E$ of $M$ has the cone property if

1. $E$ is closed in $G$,

2. for every $x \in E$ there exists an open neighbourhood $U_{x}$ of $x$ in $G$ and a $C^{\infty}$ mapping $\psi_{x}$ from an open subset $W_{x} \subset \mathbb{R}^{m}$ containing 0 into a Lie group of affine transformations $A_{x}$ on $G$ such that $\psi_{x}(0)=\mathbb{I}_{G}$ and there exists an open subset $W_{x}^{0}$ in $W_{x}$ such that

(a) 0 is in the closure of $W_{x}^{0}$,

(b) for every $y \in U_{x} \cap E, \psi_{x}\left(W_{x}^{0}\right) y$ is contained in $E$ and open in $M$ and the mapping $W_{x}^{0} \rightarrow \psi_{x}\left(W_{x}^{0}\right) y, t \mapsto \psi_{x}(t) y$, is a diffeomorphism.

REMARK 4.7. Let $B$ be a Lie group acting continuously by continuous automorphisms on our Lie group $G$. Then the group $B$ also acts continuously by continuous automorphisms on the Fourier algebra $A(G)$, since for any $b \in B$, the representation $\lambda \circ b$ is equivalent to $\lambda$.

THEOREM 4.8. Let $G$ be a Lie group such that $A(G)$ has an approximate identity, let $M$ be a smooth m-dimensional submanifold of $G$ and let $E \subset M$ be a subset with the cone property. Then $E$ is a set of smooth synthesis.

Proof. Let $T$ be an element of $J_{\mathcal{D}}(E)^{\perp}$. We must prove that $T$ annihilates $I_{A}(E)$. Since $A(G)$ has an approximate identity, we can assume that $T$ has compact support. In order to prove that $\left\langle T, I_{A}(E)\right\rangle=\{0\}$, it suffices to show that $T$ can be approximated in the weak* topology by elements $T_{\nu}=\lambda\left(m_{\nu}\right)$ of $B(E)$.

We shall show first that for every $x \in E$, there exists an open neighbourhood $U_{x}^{0}$ of $x$ contained in $U_{x}$ such that each $T \in J_{\mathcal{D}}(E)^{\perp}$ which is supported in $E \cap U_{x}^{0}$ is the weak ${ }^{*}$ limit of a sequence $\left\{T_{\nu} \in B(E)\right\}_{\nu}$. Fix $x$. To shorten the notation, we shall omit the index $x$ for neighbourhoods and mappings.

We can assume that $U=U_{x}$ is the domain of a chart $S: U \rightarrow \mathbb{R}^{n}$ for which

$$
S(M \cap U)=B \times\left\{0_{\mathbb{R}^{n-m}}\right\}
$$

and $B$ is an open subset of $\mathbb{R}^{m}$. By shrinking $W^{0}=W_{x}^{0}$ and $U$ if necessary, we can also assume that there exists an open relatively compact subset $O$ in $B$ such that $S(x) \in O \times\left\{0_{\mathbb{R}^{n-m}}\right\}$ and $\psi(t)\left(S^{-1}\left(O \times\left\{0_{\mathbb{R}^{n-m}}\right\}\right)\right) \subset M \cap U$ for all $t \in W_{x}^{0}$. For $t \in W^{0}=W_{x}^{0}$ let

$$
\phi(t): O \rightarrow B, \quad(\phi(t)(c), 0):=S\left(\psi(t)\left(S^{-1}((c, 0))\right)\right), \quad t \in W_{x}^{0}, c \in O .
$$


Now assume that $T$ is supported in $U \cap E$ and consider $T$ as a distribution on $G$. We transform it into a distribution $\widetilde{T}$ on $\mathbb{R}^{m} \times \mathbb{R}^{n-m}$ via the diffeomorphism $S$. Since the support of $T$ is contained in $U \cap M$, the support of $\widetilde{T}$ is contained in $B \times\left\{0_{\mathbb{R}^{n-m}}\right\}$ and so by the classical formula of L. Schwartz, there exist a finite collection of distributions $d^{\beta}$ on $B\left(\beta \in \mathbb{N}^{n-m}\right)$ such that for any $f \in \mathcal{D}(G)$,

$$
\langle T, f\rangle=\left\langle\widetilde{T}, f \circ S^{-1}\right\rangle=\sum_{\beta}\left\langle d^{\beta}, \partial_{2}^{\beta}\left(f \circ S^{-1}\right)(\cdot, 0)\right\rangle,
$$

where the partial derivative $\partial_{2}^{\beta}$ acts on the variable in $\mathbb{R}^{n-m}$. It follows from the properties of $T$ that $\widetilde{T}$ vanishes on every function $a$ in $\mathcal{D}\left(\mathbb{R}^{n}\right)$ which is zero on $\mathbb{R}^{m} \times\left\{0_{\mathbb{R}^{n-m}}\right\}$. Therefore, by standard arguments (see for instance $[\mathrm{KM}]) d^{\beta}=0$ for any $\beta \neq 0$ and so

$$
\begin{aligned}
\langle T, f\rangle & =\left\langle\widetilde{T}, f \circ S^{-1}\right\rangle=\left\langle d^{0},\left(f \circ S^{-1}\right)(\cdot, 0)\right\rangle \\
& =\sum_{\alpha} \int_{B} \varphi_{\alpha}(r) \partial^{\alpha}\left(f \circ S^{-1}\right)(r, 0) d r,
\end{aligned}
$$

where the $\varphi_{\alpha}$ are continuous functions and the partial derivatives $\partial^{\alpha}$ act only on the variable $r \in \mathbb{R}^{m}$.

Choose a decreasing sequence $\left(W_{\nu}\right)_{\nu}$ of open neighbourhoods of 0 in $W$ and let $W_{0, \nu}=W^{0} \cap W_{\nu}$ for all $\nu$. Since 0 is contained in the closure of $W^{0}$ we know that $W_{0, \nu} \neq \emptyset$ for $\nu$ large enough. Let $y \in O$ and let $V_{y, \nu}:=\left\{\phi(t) y: t \in W_{0, \nu}\right\}$. Then $V_{y, \nu}$ is an open subset of $B$ and the mappings $\Phi_{y, \nu}: W_{0, \nu} \rightarrow V_{y, \nu}, t \mapsto \phi(t) y$, are diffeomorphisms by the condition on $\psi$. We can assume that there exists an open subset $B_{0} \subset$ $O$ such that $S(x) \in B_{0} \times\left\{0_{\mathbb{R}^{n-m}}\right\}$, for every $\nu$ a small open set $W_{1, \nu}$ in $W_{0, \nu}$, and an open neigbourhood $C_{0}^{\nu}$ of 0 in $\mathbb{R}^{m}$, such that the open subset $O_{y, \nu}:=\left\{\phi(t)(y)+z: t \in W_{1, \nu}, y \in B_{0}, z \in C_{0}^{\nu}\right\}$ of $B$ is contained in $V_{y, \nu}$ for every $\nu \in \mathbb{N}$. For $z \in C_{0}^{\nu}$ and $y \in B_{0}$ we denote the open subset $\left\{\phi(t)(y)+z: t \in W_{1, \nu}\right\}$ of $O_{y, \nu}$ by $O_{y, \nu}^{z}$. Let $\varrho_{y, \nu}^{z}$ be the restriction to $O_{y, \nu}^{z}$ of the inverse of the mapping $W_{1, \nu} \rightarrow O_{y, \nu}^{z}, t \mapsto$ $\phi(t) y+z$.

Let now $\beta_{\nu} \in C_{\mathrm{c}}^{\infty}\left(W_{1, \nu}\right)$ such that $\beta_{\nu}(t) \geq 0$ for all $t \in W_{1, \nu}$ and $\int_{W_{1, \nu}} \beta_{\nu}(t) d t=1$. Hence for every $f \in \mathcal{D}(B), y \in B_{0}^{\nu}$ and $z \in C_{0}^{\nu}$ we have, by the change of variable formula,

$$
\int_{W_{\nu}^{0}} \beta_{\nu}(t) f(\phi(t)(y)+z) d t=\int_{O_{y, \nu}} \beta_{\nu}\left(\varrho_{y, \nu}^{z}(r)\right) \operatorname{Jac}\left(\varrho_{y, \nu}^{z}\right)(r) f(r) d r .
$$

Hence, differentiating this equation in $z$ and using again the change of variable formula for the mapping $t \mapsto \phi(t)(y)$, for every multi-index $\alpha \in \mathbb{N}^{m}$, 
we see that

$$
\begin{aligned}
\int_{W_{\nu}^{0}} \beta_{\nu}(t) \partial^{\alpha} f(\phi(t) y) d t & =\int_{O_{y, \nu}} p_{\alpha, \nu, y}(r) f(r) d r \\
& =\int_{W_{\nu}^{0}} q_{\alpha, \nu}(t, y) f(\phi(t) y) d t
\end{aligned}
$$

for some function $p_{\alpha, \nu, y} \in \mathcal{D}\left(O_{y, \nu}\right)$ which is $C^{\infty}$ in $y$, and some function $q_{\alpha, \nu}(\cdot, y)$ in $\mathcal{D}\left(W_{0, \nu}\right)$ which is also $C^{\infty}$ in $y$.

Since $\psi(t)$ is affine for every $t \in W_{0}$ and the mapping $(t, s) \mapsto \psi(t) s \in G$ is continuous (even $C^{\infty}$ ), it follows that for every $u \in A(G)$ the mapping $W_{x} \ni t \mapsto \psi(t) u$, where $\psi(t) u(x):=u(\psi(t) x), x \in G$, is also continuous, and so $u_{\nu}$ defined by

i.e.

$$
u_{\nu}:=\beta_{\nu} \cdot u:=\int_{W_{1, \nu}} \beta_{\nu}(t) \psi(t) u d t,
$$

$$
u_{\nu}(g):=\int_{W_{1, \nu}} \beta_{\nu}(t) u(\psi(t) g) d t, \quad g \in G,
$$

is an element of $A(G)$. Furthermore, since the support of $\beta_{\nu}$ is contained in $W_{1, \nu}$ and since $\psi(0)=\mathbb{I}$, we have $\lim _{\nu \rightarrow \infty} u_{\nu}=u$. This tells us also that the new elements $T_{\nu} \in \mathrm{VN}(G), \nu \in \mathbb{N}$, defined by

$$
\left\langle T_{\nu}, u\right\rangle:=\left\langle T, u_{\nu}\right\rangle=\left\langle T, \beta_{\nu} \cdot u\right\rangle
$$

converge in the weak* topology to $T$.

Let now $U_{x}^{0}:=S^{-1}\left(B_{0} \times \mathbb{R}^{n-m} \cap S\left(U_{x}\right)\right)$. Then by (4.21) and (4.23), for $f \in \mathcal{D}\left(U_{x}^{0}\right)$, we have

$$
\begin{aligned}
\left\langle T_{\nu}, f\right\rangle & =\left\langle T, f_{\nu}\right\rangle=\sum_{\alpha} \int_{B} \varphi_{\alpha}(y) \partial^{\alpha}\left(f_{\nu} \circ S^{-1}\right)(y, 0) d y \\
& =\sum_{\alpha} \int_{B} \int_{W_{1, \nu}} \varphi_{\alpha}(y) \beta_{\nu}(t) \partial^{\alpha}\left(f \circ S^{-1}\right)(\phi(t) y, 0) d t d y \\
& =\sum_{\alpha} \int_{B} \int_{W_{1, \nu}} \varphi_{\alpha}(y) q_{\alpha, \nu}(t, y)\left(f \circ S^{-1}\right)(\phi(t) y, 0) d t d y .
\end{aligned}
$$

This shows that $T_{\nu}$ is in fact a measure for every $\nu$. Furthermore if $f$ is zero on $E$, then for any $y \in S\left(U_{x}^{0} \cap E\right)$, we have

$$
0=f(\psi(t) y)=f \circ S^{-1}(\phi(t)(S(y)), 0) \quad \text { for all } t \in W_{1, \nu},
$$

since $\psi(t) y \in E$ for all $y \in U_{x}^{0}$ and $t \in W_{1, \nu}$. Hence $\left\langle T_{\nu}, f\right\rangle=0$ for all $\nu$. This implies that $\langle T, u\rangle=0$ for every $u \in A(G)$ which vanishes on $E$.

Let now $T \in J_{\mathcal{D}}(E)^{\perp}$ with compact support. Since $\operatorname{supp} T \subset E$, there exists a finite set $L$ in $E$ such that $\operatorname{supp} T \subset \bigcup_{x \in L} U_{x}^{0}$. For every $x \in L$, let $\varphi_{x} \in \mathcal{D}(G) \subset A(G)$ be such that $\operatorname{supp}\left(\varphi_{x}\right) \subset U_{x}^{0}$ and $\sum_{x \in L} \varphi_{x}=1$ on a 
compact neighbourhood $C$ of $\operatorname{supp} T$. Then $\varphi_{x} T$ is in $J_{\mathcal{D}}(E)^{\perp}$ and has its support in $U_{x}^{0} \cap E$ for every $x \in L$ and $T=\sum_{x \in L} \varphi_{x} T$. Therefore, by what we have seen above,

$$
\varphi_{x} T=w^{*} \lim \lambda\left(m_{\nu}^{x}\right) \quad \text { with } m_{\nu}^{x} \in B(E)
$$

and so $T=w^{*} \lim _{\nu} \lambda\left(\sum_{x \in L} m_{\nu}^{x}\right)$.

The following corollary generalizes the result of Kirsch-Müller in [KM].

Corollary 4.9. Let $G$ be a Lie group and let $B$ be a group of affine transformations of $G$. Let $\omega \subset G$ be a closed $m$-dimensional B-orbit in $G$. Then $\omega$ is a set of weak synthesis with $I_{A}(\omega)^{[m / 2]+1}=J_{A}(\omega)$.

Proof. Let $z \in \omega$ and let $B_{0}$ be the stabilizer of $z$ in $B$. Then $\omega \simeq B / B_{0}$ via the diffeomorphism $B / B_{0} \rightarrow \omega, t B_{0} \mapsto t(z)=: t B_{0} \cdot z$. We choose neighbourhoods $M \subset L$ in $B / B_{0}$ of $\mathbb{I}_{G}$ such that $L$ is the domain of a chart $S: L \rightarrow S(L) \subset \mathbb{R}^{m}$ (for some $m \in \mathbb{N}$ ), $\mathfrak{b}:=S(M)$ is a closed euclidean ball with centre 0 , and $S\left(\mathbb{I} \bmod B_{0}\right)=0$. We can also suppose that there exists a smooth section $\sigma: L \rightarrow B$ with $\sigma(M) M \subset L$. Furthermore, since the mapping $t \mapsto \sigma\left(S^{-1}(t)\right) \bmod B_{0}$ is regular in a neighbourhood of 0 , we can assume that $\mathfrak{b}$ is small enough so that the mappings $\mathfrak{b} \ni t \mapsto$ $\sigma\left(S^{-1}(t)\right) S^{-1}(u) \in B / B_{0}$ are regular and hence are diffeomorphisms onto their images for every $u \in \mathfrak{b}$. In the chart $S: L \rightarrow \mathbb{R}^{m}$, we can write

$$
S\left(\sigma\left(S^{-1}(v)\right) \cdot S^{-1}(u)\right)=u+R(u) v+v_{0}(u), \quad v, u \in \mathfrak{b}
$$

where $R(u)$ is an invertible linear mapping of $\mathbb{R}^{m}$ which depends smoothly on $u$ and where $v_{0}(u)$ is a vector in $\mathbb{R}^{m}$ which also varies smoothly with $u$ and is of length $o(\|v\|)\|v\|$, where \|\| denotes the euclidean norm on $\mathbb{R}^{m}$.

LEMMA 4.10. The closed subset $E_{z}=\sigma\left(S^{-1}(\mathfrak{b})\right)(z)$ of $\omega$ has the cone property.

Proof. Fix a point $x=\sigma\left(S^{-1}(u)\right)(z) \in E_{z}(u \in \mathfrak{b})$. Using (4.24) we choose a small open cone $C^{\prime}$ in $\mathbb{R}^{m}$ containing 0 in its closure, such that the radius $r$ of $\mathfrak{b}$ satisfies

$$
r-\left\|u^{\prime}+c^{\prime}\right\| \geq K\left\|c^{\prime}\right\|
$$

for all $u^{\prime}$ in an open neighbourhood $\mathfrak{k}$ of $u$ in $\mathfrak{b}$ and for all $c^{\prime} \in C^{\prime}$ (for some constant $K>0$ ), and $C:=R(u)^{-1}\left(C^{\prime}\right) \subset \mathfrak{b}$. For $c=R(u)^{-1} c^{\prime} \in C, u^{\prime} \in \mathfrak{k}$, we then have

$$
\begin{aligned}
& r-\| S\left(\sigma\left(S^{-1}(c)\right)\right.\left.\cdot S^{-1}\left(u^{\prime}\right)\right)\|=r-\| u^{\prime}+R\left(u^{\prime}\right) c+c_{0}\left(u^{\prime}\right) \| \\
& \geq r-\left\|u^{\prime}+c^{\prime}\right\|-\left\|R\left(u^{\prime}\right) R(u)^{-1}\left(c^{\prime}\right)-c^{\prime}\right\|-\left\|c_{0}\left(u^{\prime}\right)\right\| \\
& \geq K\left\|c^{\prime}\right\|-\left\|R\left(u^{\prime}\right) R(u)^{-1}\left(c^{\prime}\right)-c^{\prime}\right\|-\left\|c_{0}\left(u^{\prime}\right)\right\|>0
\end{aligned}
$$

for $\mathfrak{k}$ small enough. This implies by (4.24) that

$$
S\left(\sigma\left(S^{-1}(c)\right) \cdot S^{-1}\left(u^{\prime}\right)\right) \in \mathfrak{b}
$$


for all $u^{\prime} \in \mathfrak{k}$ and $c \in C$, if we choose $\mathfrak{k}$ small enough. Let

$$
V_{x}=: \sigma\left(S^{-1}(\mathfrak{k})\right) \cdot z=\sigma\left(S^{-1}(\mathfrak{k})\right)(z) .
$$

Then $V_{x}$ is an open subset of $E_{z}$ containing $x$. Let $U_{x}$ be any open subset in $G$ such that $U_{x} \cap E_{z}=V_{x}$. Define also

$$
W_{x}:=\mathfrak{b}, \quad W_{x}^{0}:=C \quad \text { and } \quad \psi_{x}(c):=\sigma\left(S^{-1}(c)\right) \in B, \quad c \in W_{x} .
$$

Then for every $y=\sigma\left(S^{-1}\left(u^{\prime}\right)\right)(z) \in V_{x}, u^{\prime} \in \mathfrak{k}$, by (4.25) we have

$$
\left.\psi_{x}(c)(y)=\sigma\left(S^{-1}(c)\right) S^{-1}\left(u^{\prime}\right)\right) \cdot z \in \sigma\left(S^{-1}(\beta)\right) z \in E_{z}
$$

for any $c \in C$. This shows that $E_{z}$ has the cone property.

Since $\omega$ is the union of the sets $E_{z}, z \in A$, and since being of smooth synthesis is a local property, $\omega$ is a set of smooth synthesis and so by Corollary 4.4 it is of weak synthesis with $I_{A}(\omega)^{[m / 2]+1}=J_{A}(\omega)$, which completes the proof of Corollary 4.9 .

\section{Some applications to the Varopoulos algebra $V(G)$ and linear} operator equations. Let $C(G)$ denote the algebra of continuous complexvalued functions on a compact group $G$ and let

$$
V(G)=C(G) \hat{\otimes} C(G),
$$

the projective tensor product of $C(G) . V(G)$ is the Banach algebra of complex-valued continuous functions $w$ on $G \times G$ which admit representations

$\left(w=\sum_{i=1}^{\infty} \varphi_{i} \otimes \psi_{i}\right)$ such that

$$
w(s, t)=\sum_{i=1}^{\infty} \varphi_{i}(s) \psi_{i}(t)
$$

$$
\sum_{i=1}^{\infty}\left\|\varphi_{i}\right\|_{\infty}\left\|\psi_{i}\right\|_{\infty}<\infty
$$

with the norm defined by

$$
\|w\|_{V}=\inf \left\{\sum_{i=1}^{\infty}\left\|\varphi_{i}\right\|_{\infty}\left\|\psi_{i}\right\|_{\infty}: w=\sum_{i=1}^{\infty} \varphi_{i} \otimes \psi_{i}\right\} .
$$

$V(G)$ is a semisimple regular commutative Banach algebra with spectrum $G \times G$. In [V] Varopoulos proved that if $G$ is a compact Abelian group then the question of spectral synthesis for the Fourier algebra $A(G)$ is closely related to the one for the algebra $V(G)$. This result was extended in [ST] to arbitrary compact groups $G$.

For a closed subset $E \subset G$, put $E^{*}=\left\{(t, s): s t^{-1} \in E\right\}$. Repeating mainly the arguments in [ST, Theorem 4.6] one also obtains the following 
Proposition 5.1. Let $G$ be a compact group, and $E \subset G$ a closed subset. Assume that $I_{A}(E)^{m}=J_{A}(E)$ for some $m \in \mathbb{N}^{*}$. Then $I_{V}\left(E^{*}\right)^{m}=$ $J_{V}\left(E^{*}\right)$.

In $[\mathrm{ShT}, \mathrm{LT}]$ it was shown that spectral synthesis for $A(G)$ can be applied to the study of linear operator equations. Below we will see that the results of Section 4 are also applicable to equations of this type.

Consider the space $V^{\infty}(G)$ of all (marginal equivalence classes ([A, ShT]) of) functions $w(s, t)$ that can be written in the form

$$
w(s, t)=\sum_{n=1}^{\infty} f_{n}(s) g_{n}(t)
$$

with $f_{n} \in L^{\infty}(G, m), g_{n} \in L^{\infty}(G, m)$ and

$$
\sum_{n=1}^{\infty}\left|f_{n}(s)\right|^{2} \leq C, \quad s \in G, \quad \sum_{n=1}^{\infty}\left|g_{n}(t)\right|^{2} \leq C, \quad t \in G .
$$

In tensor notations $V^{\infty}(G)=L^{\infty}(G, m) \hat{\otimes}^{w^{*} h} L^{\infty}(G, m)$, the weak ${ }^{*}$ Haagerup tensor product $([\mathrm{BSm}])$. For $w \in V^{\infty}(G)$ one defines null $w$ as the largest set (up to a marginally null set, see $[\mathrm{A}, \mathrm{ShT}]$ ) where $w$ is zero.

For $w \in V^{\infty}(G)$, we denote by $\Delta_{w}$ the operator $T \mapsto w \cdot T=\sum_{i=1}^{\infty} M_{g_{i}} T M_{f_{i}}$ on $B\left(L^{2}(G)\right.$ ) (the sum converges in the strong operator topology). For a linear operator $\Delta$ on $B\left(L^{2}(G)\right)$ the ascent, asc $\Delta$, of $\Delta$ is the least positive integer such that $\operatorname{ker}\left(\Delta^{m}\right)=\operatorname{ker}\left(\Delta^{m+1}\right)$.

Proposition 5.2. Let $G$ be a locally compact group. If $I_{A}(E)^{m}=J_{A}(E)$ for a closed subset $E \subset G$ and $m \in \mathbb{N}^{*}$ then asc $\Delta_{w} \leq m$ for any $w \in V^{\infty}(G)$ such that null $w=E^{*}$.

Proof. By [ShT, Proposition 5.1], if $w^{k} \cdot T=0$ for $k \in \mathbb{N}^{*}$ then $\operatorname{supp}(T) \subset$ null $w=E^{*}$ (see [ShT] for the definition of the support of an operator). On the other hand, under the assumptions of the proposition one can prove, using arguments similar to those in [LT], that $w^{m} \cdot T=0$ for any $T$ supported in $E^{*}$. Thus we have the inclusions

$$
\left\{T: \operatorname{supp}(T) \subset E^{*}\right\} \subset \operatorname{ker}\left(\Delta_{w}^{m}\right) \subset \operatorname{ker}\left(\Delta_{w}^{m+1}\right) \subset\left\{T: \operatorname{supp}(T) \subset E^{*}\right\},
$$

giving the statement.

Acknowledgments. This work was partially written when the first author was visiting Chalmers University of Technology in Göteborg, Sweden, and when the second author was a visiting professor at Metz University, France. The first author was also partially supported by the research grant R1F104C09 of the University of Luxembourg and the second author by the Swedish Research Council. 


\section{References}

[A] W. Arveson, Operator algebras and invariant subspaces, Ann. of Math. (2) 100 (1974), 433-532.

[BSm] D. P. Blecher and R. R. Smith, The dual of the Haagerup tensor product, J. London Math. Soc. (2) 45 (1992), 126-144.

[D] C. F. Dunkl, Functions that operate in the Fourier algebra of a compact group, Proc. Amer. Math. Soc. 21 (1969), 540-544.

[E] P. Eymard, L'algèbre de Fourier d'un groupe localement compact, Bull. Soc. Math. France 92 (1964), 181-236.

[G] K. H. Guo, A remark on the spectral synthesis property for hypersurfaces of $\mathbb{R}^{n}$, Proc. Amer. Math. Soc. 121 (1994), 185-192.

[HKKR] H. Helson, J.-P. Kahane, Y. Katznelson and W. Rudin, The functions which operate on Fourier transforms, Acta Math. 102 (1959), 135-157.

[H] C. Herz, Harmonic synthesis for subgroups, Ann. Inst. Fourier (Grenoble) 23 (1973), no. 3, 91-123.

[Ka] J.-P. Kahane, Séries de Fourier absolument convergentes, Ergeb. Math. Grenzgeb. 50, Springer, Berlin, 1970.

$[\mathrm{KM}] \quad$ W. Kirsch and D. Müller, On the synthesis problem for orbits of Lie groups in $\mathbb{R}^{n}$, Ark. Mat. 18 (1980), 145-155.

[LT] J. Ludwig and L. Turowska, On the connection between sets of operator synthesis and sets of spectral synthesis for locally compact groups, J. Funct. Anal. 233 (2006), 206-227.

[M] D. Müller, On the spectral synthesis problem for hypersurfaces of $\mathbb{R}^{N}$, ibid. 47 (1982), 247-280.

[Ri] D. Rider, Functions which operate in the Fourier algebra of a compact group, Proc. Amer. Math. Soc. 28 (1971), 525-530.

[ShT] V. Shulman and L. Turowska, Operator synthesis II. Individual operator synthesis and linear operator equations, J. Reine Angew. Math. 590 (2006), 143-187.

[ST] N. Spronk and L. Turowska, Spectral synthesis and operator synthesis for compact groups, J. London Math. Soc. (2) 66 (2002), 361-376.

[V] N. Th. Varopoulos, Tensor algebras and harmonic analysis, Acta Math. 119 (1967), 51-112.

[VSC] N. Th. Varopoulos, L. Saloff-Coste and T. Coulhon, Analysis and Geometry on Groups, Cambridge Tracts in Math. 100, Cambridge Univ. Press, 1992.

[Wa] N. R. Wallach, Harmonic Analysis on Homogeneous Spaces, Pure Appl. Math. 19, Dekker, 1973.

[W] C. R. Warner, Weak spectral synthesis, Proc. Amer. Math. Soc. 99 (1987), $244-248$.

Department of Mathematics

University of Metz

F-57045 Metz, France

E-mail: ludwig@univ-metz.fr
Department of Mathematics Chalmers University of Technology SE-412 96 Göteborg, Sweden E-mail: turowska@math.chalmers.se

Received November 20, 2005

Revised version April 27, 2006 